\title{
Micro-displacement or bending measurement using a long-period fibre grating in a self-referenced fibre optic intensity sensor
}

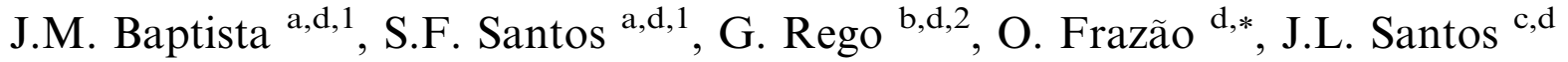 \\ a Departamento de Electrotecnia, Instituto Superior de Engenharia do Porto, Rua Dr. António Bernardino de Almeida 431, $4200-072$ Porto, Portugal \\ ${ }^{\mathrm{b}}$ Escola Superior de Tecnologia e Gestão, Instituto Politécnico de Viana de Castelo, Av. do Atlântico, 4900-348 Viana do Castelo, Portugal \\ ${ }^{\mathrm{c}}$ Departamento de Física, Faculdade de Ciências, Universidade do Porto, Rua do Campo Alegre 687, 4169-007 Porto, Portugal \\ d INESC Porto, Unidade de Optoelectrónica e Sistemas Electrónicos, Rua do Campo Alegre 687, 4169-007 Porto, Portugal
}

Received 16 March 2005; received in revised form 24 September 2005; accepted 4 October 2005

\begin{abstract}
The measurement of micro-displacement or bending using a long-period fibre grating (LPG) in a self-referenced fibre optic intensity sensor is presented. The sensing head is based on a LPG whose attenuation peak changes as a function of its micro-displacement or bending and the fibre optic intensity sensor is referenced in frequency. Two other LPGs were used as rejection filters, located at the output of the optical source to shorten its spectral width. The implemented experimental set-up is described and, the results are presented being considered the measurement range and the intrinsic resolution.
\end{abstract}

(C) 2005 Elsevier B.V. All rights reserved.

Keywords: Long-period fibre gratings; Optical fibre sensors; Optical fibre intensity sensors

\section{Introduction}

Fibre optic sensors offer the added advantages of ease and in situ measurement, flexibility, geometric versatility, ruggedness, portability, and capability of sensor multiplexing. Immunity to electrical and electromagnetic interference and resistance to corrosive and hostile environments that may comprise hazardous chemicals are also remarkable features [1].

Optical fibre intensity-modulated sensors are also very attractive since they are conceptually simple, reliable, small-sized and suitable for a wide range of applications at lower costs. However, to ensure accurate measurements, the implementation of a reference channel in the sensor is vital. Such a channel should provide insensitivity to source intensity fluctuations and to variable optical transmission

\footnotetext{
* Corresponding author. Tel.: +351 226082 601; fax: +351 226082799.

E-mail address: ofrazao@goe.fc.up.pt (O. Frazão).

1 Tel.: +351 228340 500; fax: +351 228321159 .

${ }^{2}$ Tel.: +351 258819 700; fax: +351 258827636 .
}

losses in the fibre link, couplers and connectors, which are often indistinguishable from transducer caused effects [2]. Previously, the authors reported a frequency based approach to have self-referenced fibre optic intensity sensors which are sensitive only to the losses induced by the measurand in the sensing head, i.e., the sensor readout is independent on any other optical loss that can occur along the remaining optical system [3-5].

On the other hand, long-period fibre gratings (LPGs) have been used in various types of applications, namely, bending sensing, being their measurements mainly based on the wavelength shift of the central resonance [6-11]. The problem that arises by using LPGs is the difficulty to interrogate its spectral response since the resonant bands have large bandwidth. Commonly, an optical spectrum analyzer (OSA) is used, but this equipment is unpractical for real-time applications due to its size and high cost. In this work, we present an alternative interrogation scheme. The micro-displacement or bending applied to a LPG, causes not only a wavelength shift of the central resonance but also changes its attenuation. This effect is responsible 
for the change in the transmitted optical power and constitutes the sensing parameter. The LPG sensing head is integrated in a fibre optic intensity sensor, referenced in frequency, which is immune to intensity fluctuations other than the ones caused by the LPG [3-5].

\section{Experimental set-up}

Fig. 1 presents the self-referenced fibre optic intensity sensor. It relies on a Mach-Zehnder configuration and it uses the properties of its frequency response to obtain a self-referenced signal proportional to the measurand signal.

The ratio of the value of the transfer function at an offconstructive interference frequency $\left(f_{\mathrm{OI}}\right)$ to its value at a constructive interference frequency $\left(f_{\mathrm{I}}\right)-R$ parameter depends only on the optical losses inside the fibre sensing structure (intrinsic and induced losses), being not influenced by optical power fluctuations that can occur outside the sensing head. Therefore, the modulation of the $R$ parameter provides a self-referencing scheme that makes the measurand read-out independent of possible unwanted light intensity modulation along the optical system.

For the sensing head, an LPG (\#1) was fabricated using the electric arc technique [12] such that a resonant band appeared at the central wavelength of the optical source (LED with of $\lambda_{\mathrm{c}}=1503 \mathrm{~nm}$ ). Fig. 2 shows the spectral evolution of LPG \#1, as measured by the OSA for stretched and bended positions (corresponding to a minimum and maximum of the applied micro-displacement or bending, respectively). As it can be seen, while LPG \#1 was submitted to bending, the central resonance moved towards longer wavelengths and its attenuation decreased. At the same time, another resonance appeared around $1480 \mathrm{~nm}$ and its attenuation increased with grating's bending. A similar behaviour has been observed by other authors [10,11].

To overcome this unwanted effect and to shorten the LED spectrum $(\Delta \lambda=70 \mathrm{~nm})$, two other LPGs were inserted just after the LED, in a stretched position (LPGs \#2 and \#3). LPG \#2 was chosen owing to the fact that its resonant wavelength coincide with the wavelength of the unwanted band (at $1480 \mathrm{~nm}$ ), whilst LPG \#3 was inserted in order to shorten the LED spectrum. The combined effect is shown in Fig. 3. In quantitative terms, the spectral width of the optical source was shortened from

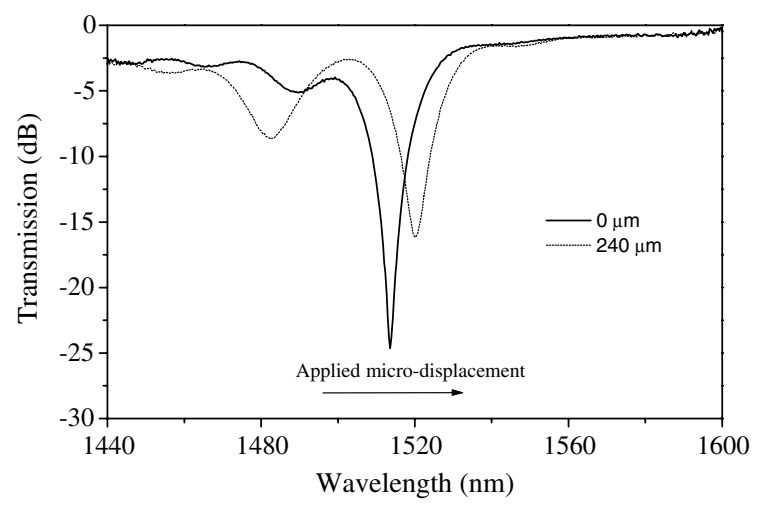

Fig. 2. Spectral evolution of LPG \#1 when subject to micro-displacement or bending.

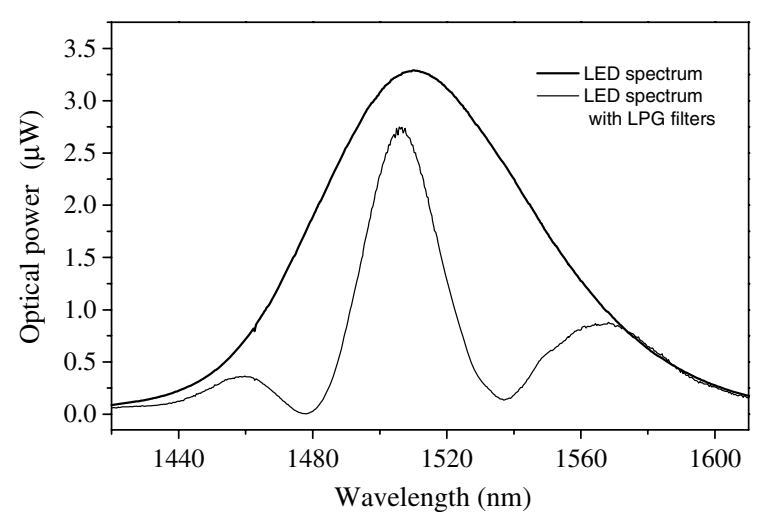

Fig. 3. Comparison of the LED spectrum with and without LPG rejection filters.

70 to $25 \mathrm{~nm}$, while the power difference across the entire spectrum rose from negligible variation to $0.25 \mathrm{~dB}$ between the two physical positions of the LPG \#1 (stretched to bended). Table 1 summarizes the characteristics of the used LPGs. It should be emphasized that this result can be improved if an optical source with a shorter spectral width is used. In present work, this was achieved by using two rejection filters. However, a better option would be the use of a band pass filter consisting of two concatenated LPGs with a core mode blocker in between [13] or the use of a $\pi$-shifted LPG [14]. On the other hand, it should be noticed that the use of a coherent source would bring

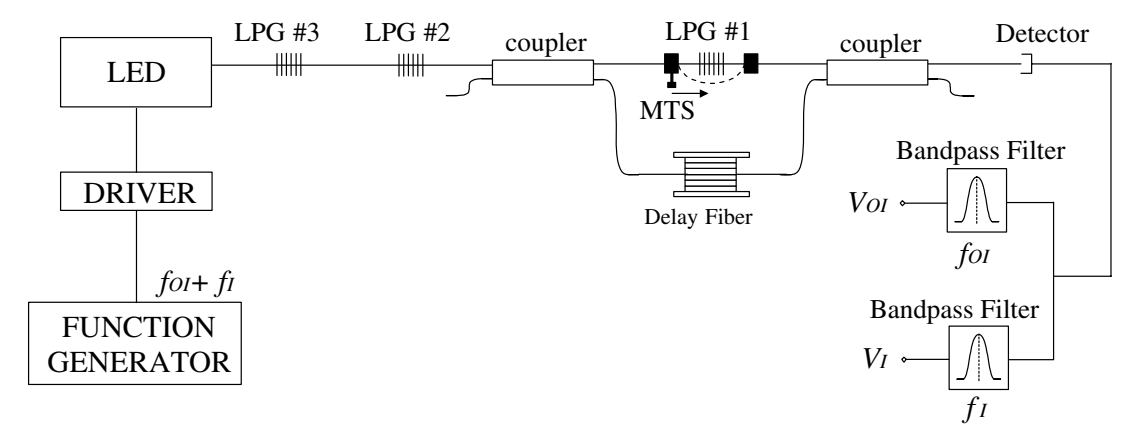

Fig. 1. Block diagram of the sensing configuration based on a Mach-Zehnder topology. 
Table 1

Characteristics of the LPGs

\begin{tabular}{lllllc}
\hline LPG & $\begin{array}{l}\text { Period } \\
(\mu \mathrm{m})\end{array}$ & $\begin{array}{l}\text { Number of } \\
\text { discharges }\end{array}$ & $\begin{array}{l}\text { Length } \\
(\mathrm{mm})\end{array}$ & $\begin{array}{l}\text { Resonant } \\
\text { wavelength }(\mathrm{nm})\end{array}$ & $\begin{array}{l}\text { Isolation } \\
\text { loss }(\mathrm{dB})\end{array}$ \\
\hline 1 & 510 & 56 & 28.0 & 1505 & -23.87 \\
2 & 500 & 36 & 17.5 & 1481 & -26.22 \\
3 & 410 & 40 & 16.0 & 1541 & -15.57 \\
\hline
\end{tabular}

polarization issues and, therefore, the requirement of more complex optical circuit, which is not desirable for this type of sensor.

To implement the self-referenced fibre optic intensity sensor shown in Fig. 1, light from a $M R V$ LED was electrically modulated by a function generator ( $H P$ model $33120 \mathrm{~A}$ ). In one arm of the Mach-Zehnder, a delay fibre of approximately $1250 \mathrm{~m}$ was inserted. Taking into account the refractive index of the fibre core, the frequency of the first constructive interference peak is of approximately $163 \mathrm{kHz}$ (not considering the one associated with case of the modulating frequency approaching zero).

In the other arm of the Mach-Zehnder topology, LPG \#1 was glued to a Micrometer Translation Stage (MTS). The distance between the two bonded extremes of LPG \#1 was $300 \mathrm{~mm}$. At the end of the Mach-Zehnder topology, light was collected by a detector followed by two narrow band pass filters whose electrical signal was analyzed by a digital oscilloscope (Gould 9500A). The level of micro-displacement or bending of the LPG is induced by the displacement of the MTS, being possible, therefore, to characterize the micro-displacement or bending measurement capacity of this sensor.

The relation between micro-displacement $(\Delta z)$ and bending $(B)$, which corresponds to the inverse of bending radius, is given as

$\Delta z=L\left[1-\frac{\sin \left(\frac{\theta}{2}\right)}{\left(\frac{\theta}{2}\right)}\right]$,

where $\sin \left(\frac{\theta}{2}\right)=\frac{L}{2} B$, being $\theta$ the arc angle and $L$ the distance between the two bonded extremes [15].

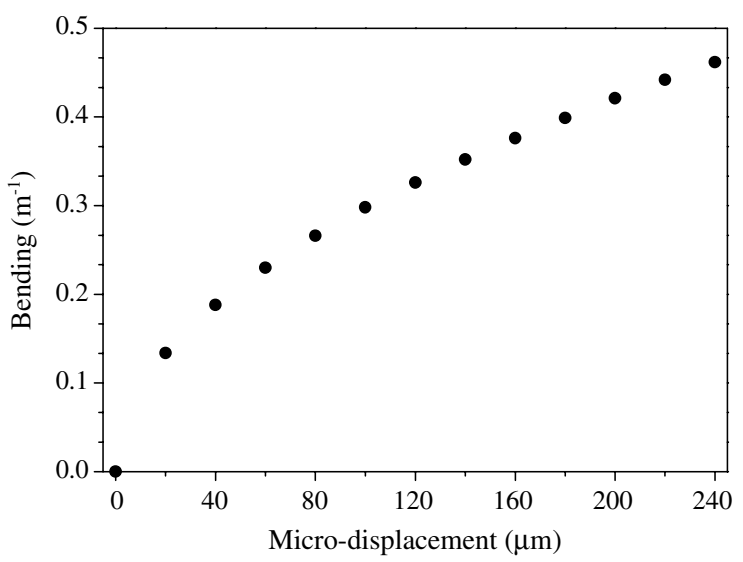

Fig. 4. Relationship between micro-displacement and bending.
Fig. 4 shows the relationship of the two physical parameters, micro-displacement vs. bending. In this case, the unit of bending is given by $\mathrm{m}^{-1}$.

\section{Results}

To observe the sensor transfer function, the electrical signal applied to the Driver was swept between 250 and $326 \mathrm{kHz}$ and the signal was taken directly from the detector. Fig. 5 displays the observed transfer function of LPG \#1, for stretched (Fig. 5(a)) and bended positions (Fig. 5(b)). The first and the second off-constructive interference frequency are clearly seen $(81.5$ and $244.5 \mathrm{kHz}$, respectively), as well as the first and the second constructive interference frequency (163 and $326 \mathrm{kHz}$, respectively).

In order to obtain the $R$ parameter measurements, two sinusoidal electrical signals with different frequencies were superimposed on the Driver. One centred in the off-constructive interference frequency $(81.5 \mathrm{kHz})$ and the other one centred in the constructive interference frequency $(163 \mathrm{kHz})$. At the detector, the two narrow band pass filters were tuned to those frequencies, respectively, being determined the rms values of the corresponding output voltage
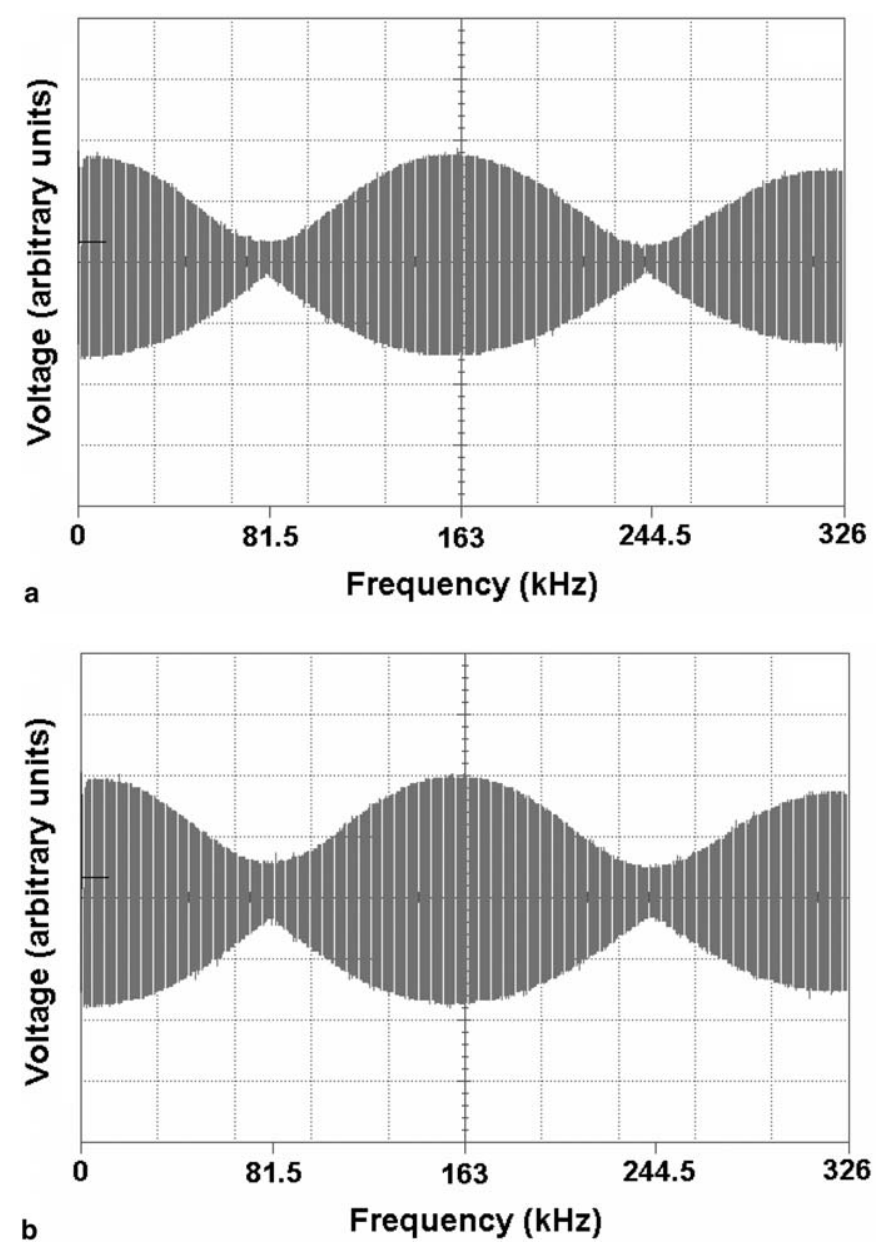

Fig. 5. Transfer function for stretched (a) and bended (b) positions of LPG \#1. 


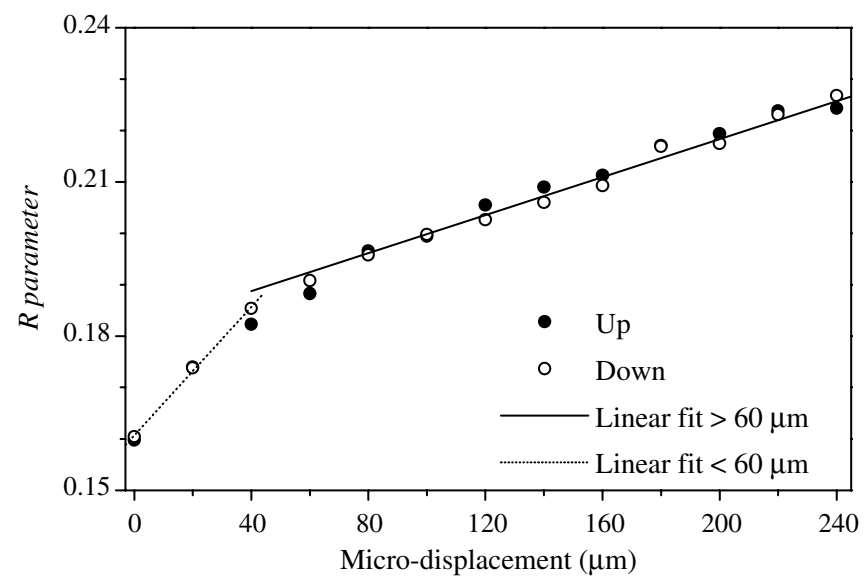

Fig. 6. Experimental $R$ parameter versus micro-displacement for an up and down measurement.

signals $\left(V_{\mathrm{OI}}\right.$ and $\left.V_{\mathrm{I}}\right)$, which are proportional to their amplitudes.

Fig. 6 shows the $R$ parameter measurement as function of an up and down micro-displacement. Step measurements of $20 \mu \mathrm{m}$ in a range of $240 \mu \mathrm{m}$ were applied. Two regions of different sensitivities can be identified: higher sensitivity in the range of $0-40 \mu \mathrm{m}$; lower sensitivity in the range of $40-240 \mu \mathrm{m}$. The two slopes results from the non linear response of the LPG (resonant wavelength and attenuation) when it is submitted to micro-displacement or bending [16]. A linear fit can be adjusted to each one of these ranges $\left(r^{2}>0.98\right)$ being the achieved resolution $\pm 11.5 \mu \mathrm{m}$. If the sensor is used as a bending sensor, the resolution for the measurement range of $0.25-0.5 \mathrm{~m}^{-1}$ is $\pm 0.02 \mathrm{~m}^{-1}$.

\section{Conclusion}

The developed fibre optic sensor demonstrated its capability of measuring micro-displacement or bending, how- ever, its performance can be enhanced using an optical source with a shorter spectral width.

The described sensing configuration requires a small number of optical components and simple signal processing. Of significant importance is the concept of self-referencing which solves the problem of the optical power fluctuations along the optical system.

Finally, this technology has the potential to be integrated in a multiplexed sensors network.

\section{References}

[1] K.T.V. Grattan, B.T. Meggitt (Eds.), Optical Fiber Sensors Technology, Kluwer Academic Publisher, 2000, p. 1.

[2] G. Murtaza, J.M. Senior, International Journal of Optoelectronics 9 (4) (1994) 339.

[3] J.M. Baptista, J.L. Santos, A.S. Lage, Optical Engineering 39 (6) (2000) 1636.

[4] J.M. Baptista, J.L. Santos, A.S. Lage, Optics Communications 181 (4-6) (2000) 287.

[5] J.M. Baptista, S. Abad, G.M. Rego, L.A. Ferreira, F.M. Araújo, J.L. Santos, A.S. Lage, Optical Engineering 43 (3) (2004) 702.

[6] S.W. James, R.P. Tatam, Measurement Science and Technology 19 (2003) 49

[7] H. Dobb, K. Kalli, D.J. Webb, Electronics Letters 40 (11) (2004) 657.

[8] S. Chen, Z. Tong, Q. Zhao, Z. Liu, X. Dong, Sensors and Actuators A 116 (2004) 103.

[9] H.J. Patrick, C.C. Chang, S.T. Vohra, Electronics Letters 34 (18) (1998) 1773.

[10] Y. Liu, L. Zhang, J.A.R. Williams, I. Bennion, IEEE Photonics Technology Letters 12 (5) (2000) 531.

[11] C.C. Ye, S.W. James, R.P. Tatam, Optics Letters 25 (14) (2000) 1007.

[12] G. Rego, O. Okhotnikov, E. Dianov, V. Sulimov, Journal of Lightwave Technology 19 (10) (2001) 1574.

[13] D.S. Starodubov, V. Grubsky, J. Feinberg, IEEE Photonics Technology Letters 10 (11) (1998) 1590.

[14] G. Humbert, A. Malki, Electronics Letters 39 (21) (2003) 1506.

[15] C.S. Goh, M.R. Mokhtar, S.A. Butler, Y. Set, K. Kikushi, M. Ibsen, IEEE Photonics Technology Letters 15 (4) (2003) 557.

[16] Y.G. Han, B.H. Lee, W.T. Han, U.C. Paek, Y. Chung, Measurement Science and Technology 12 (2001) 778. 